\title{
Nutritional supplements for age-related macular degeneration
}

\author{
Nupura Krishnadev, Annal D. Meleth, and Emily Y. Chew
}

\section{Abstract}

Purpose of review-Age-related macular degeneration, a leading cause of visual loss in older adults, has limited therapeutic options. This review describes the current literature on the role of nutritional supplementation in primary and secondary prevention of AMD.

Recent findings-Many observational studies have explored the association between diet, nutrient intake, and AMD. In particular, high dietary intakes of omega 3 fatty acids, and macular xanthophylls lutein and zeaxanthin have been associated with a lower risk of prevalent and incident AMD. However, the Age-Related Eye Disease Study (AREDS) is the only large-scale randomized controlled clinical trial to show a $25 \%$ beneficial effect of nutritional supplementation in reducing the risk progression to advanced AMD in patients with intermediate AMD or with advanced AMD in one eye at 5 years of follow-up. Based on the results of AREDS, these patients are recommended to take AREDS formulation of vitamins $\mathrm{C}, \mathrm{E}$, beta-carotene, and zinc with copper.

Summary-At the present time, there is insufficient evidence in the literature to recommend routine nutritional supplementation in healthy adults for primary prevention of AMD. However, patients with intermediate risk of AMD or advanced AMD in one eye should consider taking AREDS-type supplements. Observational studies have also suggested benefit from increased dietary intake of macular xanthophylls and omega- 3 fatty acids. These are currently being evaluated prospectively in a randomized controlled clinical trial, the Age-Related Eye Disease Study 2 (AREDS2).

\section{Keywords}

age-related macular degeneration; antioxidant vitamins; omega-3 fatty acids; lutein; zeaxanthin

\section{Introduction}

\begin{abstract}
Age-related macular degeneration (AMD) is the leading cause of visual loss in older adults in the United States and developed countries [1]. Epidemiological studies over the last two decades have provided insights into the risk factors associated with AMD, including age, sex, diet, nutritional status, smoking, hypertension, and genetic markers. While anti-vascular endothelial growth factor agents have resulted in a remarkable beneficial effect in reducing the risk of moderate vision loss in patients with neovascular AMD [2,3], a large proportion of patients with AMD still continue to lose vision, and there is no effective treatment available for the atrophic form of AMD. Therapies that focus on prevention through optimization of modifiable risk factors such as diet and nutritional status are key approaches to reducing the burden of disease. Methods that help to prevent development or progression of AMD through nutritional supplementation will become especially important as our
\end{abstract}

Corresponding Author: Dr. Emily Y. Chew, Corresponding Author's Institution: National Eye Institute, National Institutes of Health, Building 10, CRC, Room 3-2531, 10 Center Drive, MSC-1204, Bethesda, MD, 20892-1204, USA, Phone: 3014966583. echew@nei.nih.gov.

Disclosures: None 
population increases in longevity and the number of individuals affected with AMD continues to rise.

\section{Literature Review}

The literature search to support this review was performed between October 1, 2009 and November 23, 2009. Databases searched included Medline, Pubmed, Scopus, EMBASE, The Cochrane Library, and Google Scholar. English language articles published in 2008 and 2009 were reviewed, and the bibliographies of relevant publications were scanned for additional randomized controlled clinical trials.

The majority of the literature on diet and nutritional supplementation in AMD published within the last 12 to 18 months focuses on the efficacy of omega 3 fatty acids, macular xanthophylls lutein and zeaxanthin, vitamin B12 and folic acid, and the constituents of the Age-Related Eye Disease Study (AREDS) formulation, namely vitamins C, E, betacarotene, and zinc with copper. In particular, macular xanthophylls and long chain fatty acids have received significant attention in the literature since the last review on this subject in this journal in 2007 [4].

\section{AREDS and AREDS-Type Formulations}

AREDS was the first large scale randomized controlled clinical trial to demonstrate the benefit of vitamin and mineral supplementation in preventing progression to advanced AMD. The AREDS formulation of vitamin C $500 \mathrm{mg}$, vitamin E $400 \mathrm{IU}$, beta-carotene 15 $\mathrm{mg}$ and zinc (zinc oxide $80 \mathrm{mg}$ and cupric oxide $2 \mathrm{mg}$ ) showed a $25 \%$ risk reduction in progression to advanced AMD over 5 years in patients with intermediate AMD (extensive intermediate drusen in one or both eyes, one or more large drusen in at least one eye, or nonsubfoveal geographic atrophy in one eye) or advanced AMD (subfoveal geographic atrophy or choroidal neovascular membrane) in one eye [5]. The risk of losing vision of three or more lines (doubling of the visual angle) also was reduced by $19 \%$ with this combination treatment. In fact, when available nutritional studies were reviewed by the Cochrane collaboration, the main evidence regarding the effectiveness of vitamin supplementation in preventing AMD progression was attributed as deriving primarily from AREDS [6].

A recent report from the Blue Mountain Eye Study [7], a population-based study, supported the AREDS finding of a beneficial effect of zinc in AMD progression. Of the original cohort, 2454 patients were re-examined with stereoscopic fundus photography 5 and 10 years after initial study enrolment, and energy-adjusted nutrient intakes were assessed. In threshold analyses, patients in the top decile of total zinc intake $(\geq 15.8 \mathrm{mg} /$ day $)$ were significantly less likely to develop any AMD (relative risk [RR], 0.56; 95\% confidence interval [CI], 0.32-0.97) or early AMD (RR, 0.54; 95\% CI, 0.30-0.97) when compared with the remaining population. Unlike AREDS, however, the Blue Mountain Eye study also found that higher beta carotene intake was associated with an increased risk of neovascular AMD, even after adjusting for smoking status. The authors suggested that this result be taken with caution as the cause for this negative association is not known. It is also important to note that this was an observational study, and that the number of cases with advanced AMD was limited.

While the AREDS demonstrated protective benefits of supplementation in delaying AMD progression, the role of vitamins and minerals in the primary prevention of AMD is more difficult to ascertain. The AREDS formulation showed no effect in preventing the development of large drusen in participants who had small drusen at baseline, and the incidence of advanced AMD in this group was exceeding low $(<1 \%)$. In 2005, the Rotterdam Study [8] suggested that high dietary intake of beta-carotene, vitamins C and E, 
and zinc was associated with a 35\% reduction in incident AMD risk in elderly persons. More recently, the Women's Health Study randomized 39876 women were randomized to low dose aspirin and vitamin E. After 10 years of treatment and follow-up, aspirin therapy had no large or harmful effect on risk of AMD [9], with 111 cases of AMD in the aspirin group and 134 cases in the placebo group (HR, 0.82; 95\% CI, 0.64-1.06).

The Physicians Health Study II may shed some further insights into the role of vitamins C, $\mathrm{E}$, and beta carotene supplementation in preventing AMD. In this study, 14642 men were randomized to one of 16 possible combinations of vitamin C (500 mg), vitamin E (400 IU), beta-carotene $(50 \mathrm{mg})$, and a multivitamin to assess their role in the primary prevention of cardiovascular disease, cancer, cataract, and AMD [10]. Follow-up was completed in December 2007 and data analysis is in progress.

Despite these results, a recent Cochrane library review [11•] of three large scale clinical trials randomizing 23000 patients did not show a benefit to supplementation with betacarotene or vitamin $\mathrm{E}$ in preventing AMD. The review concluded that there was insufficient evidence to recommend routine supplementation with antioxidant vitamins or minerals in healthy adults to delay or prevent AMD onset.

\section{Omega-3 Long Chain Polyunsaturated Fatty Acids}

Omega 3 polyunsaturated fatty acids include alpha-linolenic acid (short chain), docosahexaenoic acid (DHA), and eicosapentaenoic acid (EPA) (both long chain). Diet is the only source of these fatty acids as they cannot be synthesized by humans de novo. DHA is present in high concentrations in the outer segments of photoreceptors, which are constantly shed and turned over during the visual cycle. Both alpha-linolenic acid and EPA are dietary precursors to DHA. Deficiencies of DHA have been implicated in AMD onset [12], and long chain omega 3 fatty acids may also help to prevent the oxidative, inflammatory and age-related retinal damage that occurs during AMD development [13].

Several epidemiological studies have suggested an inverse relationship between dietary omega-3 long-chain polyunsaturated fatty acid or fish intake and risk of AMD. In the Blue Mountain Eye Study [14•], one serving of fish per week was associated with reduced 10 year risk of incident early AMD (RR, $0.69 ; 95 \%$ CI, 0.49-0.98). This benefit was primarily among participants with less than the median baseline alpha-linoleic acid consumption. Another large Australian cohort study also showed that higher omega-3 fatty acid intake was inversely associated with early AMD when the highest and lowest quartiles were compared (odds ratio [OR],0.85; 95\% CI, 0.71-1.02) [15].

In the AREDS population, higher intakes of DHA and EPA were associated with a lower risk of progression to advanced AMD, independent of AREDS supplementation [16]. AREDS participants with highest intake of omega-3 long-chain polyunsaturated fatty acids were approximately half as likely to have neovascular AMD at baseline $(\mathrm{OR}, 0.61 ; 95 \% \mathrm{CI}$, 0.41-0.90) or to progress over a six year period from bilateral drusen to central geographic atrophy (OR, 0.44; 95\% CI, 0.23-0.87) when compared with those who had the lowest intake (AREDS report \#20 and 23) [17,18•]. In addition, a nested cohort study of AREDS patients at moderate-to-high risk of advanced AMD demonstrated a 12 year incidence of advanced AMD that was 30\% lower in patients reporting the highest consumption of omega-3 fatty acids [19].

A recent meta-analysis by Chong et al. [20 ${ }^{\circ}$ reviewed nine studies with a total sample of 88 974 people, including 3203 AMD cases (1847 early and 1356 late AMD cases). A high dietary intake of omega-3 fatty acids was associated with a $38 \%$ reduction in the risk of late AMD (pooled OR, 0.62; 95\% CI, 0.48-0.82). A minimum bi-weekly fish intake was 
associated with a reduced risk of both early AMD and late AMD. Based on the observational data reviewed, the authors concluded that consumption of omega-3 fatty acids may be associated with a lower risk of AMD but that there was insufficient evidence to recommend omega-3 fatty acid supplemention for AMD prevention in the general population. However, randomized trials are needed to test the efficacy of this nutritional factor.

\section{Lutein and Zeaxanthin}

Macular pigment is composed primarily from the xanthophylls lutein and zeaxanthin, also members of the carotenoid family. Their antioxidant properties, as well as their ability to filter short-wavelength light, may help to protect the outer retina and retinal pigment epithelium from oxidative stress and aid in cell membrane stability [21]. Interestingly, Parisi et al. noted improvement in multi-focal ERG results in $15 \mathrm{AMD}$ patients given oral supplementation with lutein and zeaxanthin as compared to age-matched controls [22].

The association between AMD risk and lutein and zeaxanthin supplementation has been explored in several large-scale epidemiological studies. The Eye Disease Case Control Study found that the risk for advanced AMD was reduced by $43 \%$ in participants in the highest quintile of dietary carotenoid intake, when compared with those in the lowest quintile (OR, 0.57; 95\% CI, 0.35-0.92) [23]. In 2006, the Carotenoids in Age-Related Macular Degeneration Study (CAREDS) concluded that lutein- and zeaxanthin-rich diets may protect against intermediate AMD in female patients less than 75 years of age [24]. More recently, the Blue Mountain Eye Study [7] reported that higher dietary lutein and zeaxanthin intake reduced the risk of incident AMD over 5 and 10 years. Participants in the top tertile of intake ( $\geq 942 \mu \mathrm{g} /$ day) had a decreased risk of incident neovascular AMD (RR, $0.35 ; 95 \% \mathrm{CI}, 0.13-0.92)$, and those with above median intakes (743 $\mu \mathrm{g})$ had a reduced risk of indistinct soft or reticular drusen when compared with the remaining population. In AREDS, dietary lutein/zeaxanthin intake (as determined by a food frequency questionnaire at enrollment) was inversely associated with prevalent neovascular AMD (OR, 0.65; 95\% CI, 0.45-0.93), geographic atrophy (OR, $0.45 ; 95 \% \mathrm{CI}, 0.24-0.86$ ), and large or extensive intermediate drusen $(\mathrm{OR}, 0.73 ; 95 \% \mathrm{CI}, 0.56-0.96)$ when the highest versus lowest quintiles were compared [25].

Measurement of macular pigment optical density (MPOD) provides a non-invasive measurement of retinal lutein and zeaxanthin. This may helpful in evaluating the association of the macular pigment with various aspects of the serum carotenoids, although the causal relationship may be difficult to elucidate. While studies have shown that MPOD is related to dietary intake or serum levels of lutein and zeaxanthin [26], the results relating MPOD to AMD have been inconsistent across populations, and are influenced by the many factors that affect uptake and distribution of these carotenoids in the body. A recent report from the Carotenoids in Age-Related Eye Disease Study (CAREDS) Group could not find a consistent cross-sectional association between MPOD and AMD [21], and suggested that prospective studies were needed to further explore this relationship.

\section{B Vitamins}

Homocysteine is an intermediary amino acid formed during metabolism of the essential amino acid methionine. Hyperhomocysteinemia, defined as a plasma homocysteine concentration of greater than $2.0 \mathrm{mg} / \mathrm{L}$ or $15 \mu \mathrm{mol} / 1$, is thought to induce vascular endothelial dysfunction, a process that has also been implicated in neovascular AMD [27]. Cross-sectional and case-control studies in the last few years suggest an association exists between elevated serum homocysteine levels and the risk of AMD [28-30]. In the Blue Mountain Eye Study [28], serum homocysteine greater than $15 \mu \mathrm{mol} / \mathrm{l}$ was associated with 
an increased likelihood of AMD in participants under 75 years of age, and in patients with serum homocysteine $\leq 15 \mu \mathrm{mol} / \mathrm{l}$, low serum B12 was associated with nearly four-fold higher odds of AMD.

Vitamin B12 and folate act as essential coenzymes during homocysteine metabolism; treatment with folic acid, vitamin B6 (pyridoxine hydrochloride) and vitamin 12 (cyanocobalamin) has shown to reduce homocysteine levels [31]. The Women's Antioxidant and Folic Acid Cardiovascular Study (WAFACS), a randomized trial, assessed whether treatment with vitamins B6 and B12 and folic acid could prevent cardiovascular events in women with pre-existing cardiovascular disease or multiple risk factors [32]. While no benefit was found in terms of cardiovascular disease reduction, the study provided an opportunity to evaluate AMD risk. Of the 5442 participants enrolled in the study, 5205 did not have a baseline diagnosis of AMD and were included the AMD analysis. The results, based on an average of 7.3 years of treatment and follow-up, indicate that those assigned to active treatment had a statistically significant $35 \%$ to $40 \%$ decreased risk of developing AMD. However, the authors suggest that the results be interpreted cautiously as AMD diagnosis was self-reported, and that findings in this group of females at increased risk of cardiovascular disease may not be applicable to the general population. While there were only a small number of cases of advanced AMD (19 cases, 17 exudative in nature, and 2 with geographic atrophy), this randomized trial does suggest that further investigation into the role of B vitamins in AMD risk is warranted.

\section{Future Directions}

The available observational and small trial data are compelling, and further research with large-scale prospective randomized controlled trials are needed to examine the role of nutritional supplementation in AMD. The National Eye Institute developed the AREDS2 trial, a multi-center, randomized trial, to assess the effects of daily oral supplementation of lutein, zeaxanthin, and/or DHA and EPA on the progression to advanced AMD, as compared to placebo (Table 1). More than 4000 participants aged 50 to 85 years were enrolled, and will be followed for 5 years. Included are participants with bilateral large drusen or large drusen in one eye with advanced AMD (neovascular AMD or central geographic atrophy) in the fellow eye at baseline. The primary outcome is the development of advanced AMD with secondary outcome of moderate visual acuity loss.

In addition, AREDS2 provides an opportunity to further refine the original AREDS formulation by eliminating beta-carotene and lowering the dose of zinc. Beta-carotene is not found in the eye but was thought to be important as a systemic antioxidant in the original AREDS formulation. Two large controlled randomized trials demonstrated an increased risk of lung cancer in smokers taking betacarotene. The high doses of zinc may not be necessary, as recent data has suggested that the systemic absorption of zinc is limited to about $25 \mathrm{mg}$ per day. For these reasons, the AREDS formulation will be tested by in a secondary randomization by assigning consenting participants to one of these four formulations (Table 2).

The socioeconomic benefits of primary and secondary prevention of AMD are enormous. In 2004, the direct medical cost of AMD treatment was estimated at \$575 million in the United States, excluding nursing home and home health care costs, and productivity losses [33]. This figure will only continue to rise as the number of individuals affected with AMD increases in parallel with the aging population, and as the expense of therapies such as antivascular endothelial growth factor (VEGF) treatments for neovascular AMD increases. It has been reported that the projected increase in cases of visual impairment and blindness from AMD by the year 2050 may be lowered by $17.6 \%$ if vitamin prophylaxis at early AMD 
presentation is given in addition to standard neovascular AMD treatment (laser or antiVEGF injections), when compared to neovascular AMD treatment alone [34••]. At an approximate cost of $\$ 100$ per patient per year, supplementation with vitamins and minerals may be a cost-effective method of therapy for patients with AMD to reduce future impairment and disability.

\section{Conclusions}

At the present time, there is insufficient evidence in the literature to recommend routine nutritional supplementation for primary prevention of AMD. However, patients with intermediate risk of AMD or advanced AMD in one eye are recommended to take AREDStype supplements, as this formulation has been proven to reduce the risk of progression to advanced AMD by $25 \%$ over 5 years. Many observational studies have also suggested benefit from increased dietary intake of additional nutrients such as carotenoids and omega-3 fatty acids. These supplements are currently being evaluated in the AREDS2 trial, a randomized controlled clinical trial testing the effects of lutein and omega-3 fatty acids on rates of the progression to advanced AMD. Although vitamin B supplementation appears to have potentially beneficial in the treatment of AMD in a randomized trial, no recommendations can be made until further studies are conducted. The cost-effectiveness of prevention with oral supplements has been demonstrated in the AREDS study. The results of AREDS2 and other studies may provide further insight into the prevention of AMD.

\section{References and Recommended Reading}

Papers of particular interest, published within the annual period of review, have been highlighted as:

- of special interest

$\bullet$ of outstanding interest

1. Congdon N, O'Colmain B, Klaver CC, et al. The Eye Diseases Prevalence Research Group. Causes and prevalence of visual impairment among adults in the United States. Arch Ophthalmol 2004;122:477-485. [PubMed: 15078664]

2. Rosenfeld PJ, Brown DM, Heier JS, et al. the MARINA Study Group. Ranibizumab for Neovascular Age-Related Macular Degeneration. NEJM 2006;355:1419-1431. [PubMed: 17021318]

3. Brown DM, Kaiser PK, Michels M, et al. the ANCHOR Study Group. Ranibizumab versus Verteporfin for Neovascular Age-Related Macular Degeneration. NEJM 2006;355:1432-1444. [PubMed: 17021319]

4. Coleman H, Chew E. Nutritional supplementation in age-related macular degeneration. Curr Opin Ophthalmol 2007;18:220-223. [PubMed: 17435429]

5. The Age-Related Eye Disease Study Research Group. A randomized, placebo-controlled, clinical trial of high-dose supplementation with vitamins $\mathrm{C}$ and $\mathrm{E}$, beta carotene, and zinc for age-related macular degeneration and vision loss. Arch Ophthalmol 2001;119:1417-36. [PubMed: 11594942]

6. Evans JR. Antioxidant vitamin and mineral supplements for slowing the progression of age related macular degeneration. Cochrane Database of Syst Rev 2006;2:CD000254. [PubMed: 16625532]

7. Tan JSL, Wang JJ, Flood V, et al. Dietary antioxidants and the long term incidence of age-related macular degeneration: The Blue Mountain Eye Study. Ophthalmology 2008;115:334-341. [PubMed: 17664009]

8. van Leeuwen R, Boekhoorn S, Vingerling JR, et al. Dietary intake of antioxidants and risk of agerelated macular degeneration. JAMA 2005;294:3101-3107. [PubMed: 16380590] 
9. Christen WG, Glynn RJ, Chew EY, et al. Low-dose aspirin and medical record-confirmed agerelated macular degeneration in a randomized trial of women. Ophthalmology 2009;116:23862392. [PubMed: 19815293]

10. Christen WG, Gaziano JM, Hennekens CH. Design of Physicians' Health Study II -- A Randomized Trial of Beta-Carotene, Vitamins E and C, and Multivitamins, in Prevention of Cancer, Cardiovascular Disease, and Eye Disease, and Review of Results of Completed Trials. Ann Epidemiol 2000;10:124-134.

11 . Evans JR, Henshaw KS. Antioxidant vitamin and mineral supplements for preventing age-related macular degeneration. Cochrane Database of Syst Rev 2008;1:CD000253. [PubMed: 18253971] This review assesses all the available, clinical trials on nutritional supplementation in the primary prevention of AMD, concluding that there is insufficient evidence to recommend routine supplementation in healthy adults to prevent AMD.

12. Bazan NG. The metabolism of omega-3 polyunsaturated fatty acids in the eye: the possible role of docosahexaenoic acid and docosanoids in retinal physiology and ocular pathology. Prog Clin Biol Res 1989;312:95-112. [PubMed: 2529559]

13. SanGiovanni JP, Chew EY. The role of omega-3 long-chain polyunsaturated fatty acids in health and disease of the retina. Prog Retin Eye Res 2005;24:87-138. [PubMed: 15555528]

14•. Tan JSL, Wang JJ, Flood V, Mitchel Pl. Dietary fatty acids and the 10-year incidence of agerelated macular degeneration: The Blue Mountain Eye Study. Arch Ophthalmol 2009;127:656665. [PubMed: 19433717] This study provides evidence of protection against early AMDfrom regularly eating fish, and greater consumption of omega-3 polyunsaturated fatty acids.

15. Chong EWT, Robman LD, Simpson JA, et al. Fat consumption and its association with age-relate macular degeneration. Arch Ophthalmol 2009;127:674-680. [PubMed: 19433719]

16. Chiu CJ, Klein R, Milton RC, et al. Does eating particular diets alter the risk of age-related macular degeneration in users of the Age-Related Eye Disease Study supplements? Br J Ophthalmol 2009;93:1241-1246. [PubMed: 19508997]

17. SanGiovanni JP, Chew EY, Clemons TE, et al. Age-Related Eye Disease Study Research Group. The relationship of dietary lipid intake and age-related macular degeneration in a case-control study: AREDS report No. 20. Arch Ophthalmol 2007;125:671-679. [PubMed: 17502507]

18•. SanGiovanni JP, Chew EY, Agrón E, et al. Age-Related Eye Disease Study Research Group. The relationship of dietary omega-3 long-chain polyunsaturated fatty acid intake with incident agerelated macular degeneration: AREDS report No. 23. Arch Ophthalmol 2008;126:1274-1279. [PubMed: 18779490] The results of this study from the AREDS group suggest that dietary omega-3 fatty acid intake is associated with a decreased risk of progression from bilateral drusen to central geographic atrophy.

19. SanGiovanni JP, Agrón E, Meleth AD, et al. \{omega\}-3 Long-chain polyunsaturated fatty acid intake and 12-y incidence of neovascular age-related macular degeneration and central geographic atrophy: AREDS report 30, a prospective cohort study from the Age-Related Eye Disease Study. Am J Clin Nutr 2009;90:1601-1607. [PubMed: 19812176]

20•. Chong EW, Kreis AJ, Wong TY, et al. Dietary omega-3 fatty acid and fish intake in the primary prevention of age-related macular degeneration: a systematic review and meta-analysis. Arch Ophthalmol 2008;126:826-833. [PubMed: 18541848] This detailed meta-analysis suggests that consumption of fish and foods rich in omega-3 fatty acids may be associated with a lower risk of AMD.

21. LaRowe TL, Mares JA, Snodderly M, et al. CAREDS Macular Pigment Study Group. Macular pigment density and age-related maculopathy in the Carotenoids in Age-Related Eye Disease Study: an ancillary study of the Women's Health Initiative. Ophthalmology 2008;115:876-883. [PubMed: 17868874]

22. Parisi V, Tedeschi M, Gallinaro G, et al. Carotenoids and antioxidants in the age-related maculopathy Italian study: multifocal electroretinogram modifications after 1 year. Ophthalmology 2008;115:324-333. [PubMed: 17716735]

23. Seddom JM, Ajani UA, Sperduto RD, et al. Dietary carotenoids, vitamins A, C, and E, and advanced age-related macular degeneration. Eye Disease Case-Control Study Group. JAMA 1994;272:1413-20. [PubMed: 7933422] 
24. Moeller SM, Parekh N, Tinker L, et al. CAREDS Research Study Group. Associations between intermediate age-related macular degeneration and lutein and zeaxanthin in the Carotenoids in Age-related Eye Disease Study (CAREDS): ancillary study of the Women's Health Initiative. Arch Ophthalmol 2006;124:1151-1162. [PubMed: 16908818]

25. Age-Related Eye Disease Study Research Group. The relationship of dietary carotenoids and vitamin $\mathrm{A}, \mathrm{E}$, and $\mathrm{C}$ intake with age-related macular degeneration in a case-control study: AREDS report no. 22. Arch Ophthalmol 2007;125:1225-1232. [PubMed: 17846363]

26. Mares JA, LaRowe TL, Snodderly DM, et al. CAREDS Macular Pigment Study Group and Investigators. Predictors of optical density of lutein and zeaxanthin in retinas of older women in the Carotenoids in Age-related Eye Disease Study (CAREDS): ancillary study of the Women's Health Initiative. Am J Clin Nutr 2006;84:1107-1122. [PubMed: 17093164]

27. Axer-Siegel R, Bourla D, Ehrlich R, et al. Association of neovascular age-related macular degeneration and hyperhomocysteinemia. Am J Ophthalmol 2004;137:84-89. [PubMed: 14700648]

28. Rochtchina E, Wang JJ, Flood VM, Mitchell P. Elevated serum homocysteine, low serum vitamin B12, folate, and age-related macular degeneration: the Blue Mountains Eye Study. Am J Ophthalmol 2007;143:344-346. [PubMed: 17258528]

29. Kamburoglu G, Gumus K, Kadayifcilar S, Eldem B. Plasma homocysteine, vitamin B12 and folate levels in age-related macular degeneration. Graefes Arch Clin Exp Ophthalmol 2006;244:565569. [PubMed: 16163497]

30. Seddon JM, Gensler G, Klein ML, Milton RC. Evaluation of plasma homocysteine and risk of agerelated macular degeneration. Am J Ophthalmol 2006;141:201-203. [PubMed: 16387004]

31. Homocysteine Lowering Trialists' Collaboration. Dose-dependent effects of folic acid on blood concentrations of homocysteine: a meta-analysis of the randomized trials. Am J Clin Nutr 2005;82:806-812. [PubMed: 16210710]

32. Christen WG, Glynn R, Chew EY, et al. Folic acid, pyridoxine, and cyanocobalamin combination treatment and age-related macular degeneration in women. The Women's Antioxidant and Folic Acid Cardiovascular Study. Arch Intern Med 2009;169:335-341. [PubMed: 19237716]

33. Rein DB, Zhang P, Wirth KE, et al. The economic burden of major adult visual disorders in the United States. Arch Ophthalmol 2006;124:1754-1760. [PubMed: 17159036]

34••. Rein DB, Wittenborn JS, Zhang X, Honeycutt AA, Lesesne SB, Saaddine J, the Vision Health Cost-Effectiveness Study Group. Forecasting age-related macular degeneration through the year 2050: the potential impact of new treatments. Arch Ophthalmol 2009;127:533-540. [PubMed: 19365036] Using statistical models based on current knowledge and trends, this report forecasts AMD and its consequences in the United States through the year 2050 with different treatment scenarios, concluding that prevalence of AMD will increase substantially by 2050 , but the use of new therapies may mitigate its effects. 
Table 1

Nutrients and doses to be tested in the primary randomization of the AREDS2 trial

\begin{tabular}{l|c}
\hline Randomization Agents & Daily Dose \\
\hline I Placebo & - \\
\hline Lutein/zeaxanthin & $10 \mathrm{mg} / 2 \mathrm{mg}$ \\
\hline DHA/EPA & $350 \mathrm{mg} / 650 \mathrm{mg}$ \\
\hline Lutein/zeaxanthin + DHA/EPA & $10 \mathrm{mg} / 2 \mathrm{mg}+350 \mathrm{mg} / 650 \mathrm{mg}$ \\
\hline
\end{tabular}




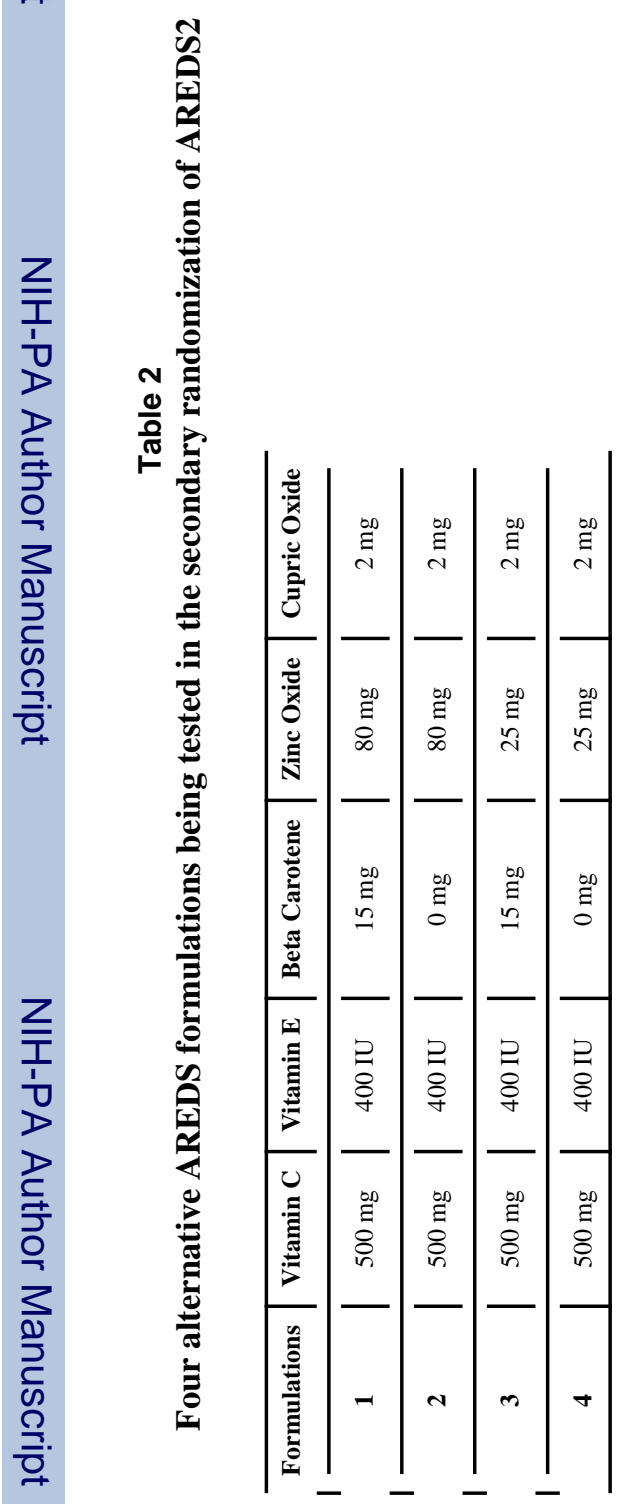

